\title{
Interprétariat communautaire et soins aux personnes «invisibles»
}

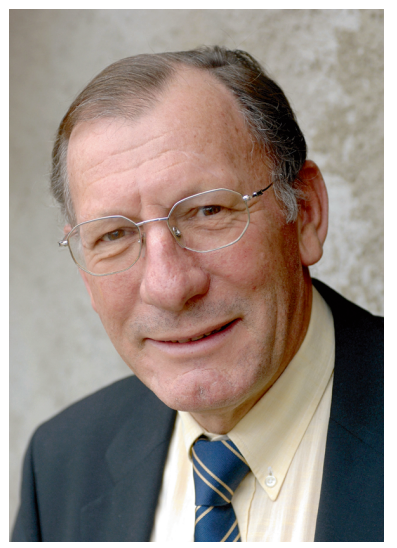

Jean Martin*
L'éclairage porté par l'article de Monika Joss [1] est très bienvenu, spécialement dans notre journal professionnel. Je le relève en tant que membre du comité d'Appartenances (www. appartenances.ch), association qui dans le canton de Vaud - à Lausanne, Vevey et Yverdon offre depuis une quinzaine d'années un éventail de services à l'intention des personnes venues d'ailleurs. Dans des registres divers: prestations psychothérapeutiques, formation élémentaire des adultes et formation continue de professionnels, promotion, animations et soutiens divers (espaces de rencontre), cours (français, informatique, couture, etc.).

Une activité importante de l'association est la formation et la mise à disposition d'interprètes communautaires. Ces derniers sont sollicités principalement dans les domaines de la santé, de l'éducation et du social, à l'intérieur d'Appartenances comme à l'extérieur (hôpitaux, écoles, services sociaux). Durant l'année dernière, il s'est agi de 17000 heures d'interprète.

A l'occasion du $10^{\mathrm{e}}$ anniversaire de ce service, Appartenances a organisé à fin 2007 une manifestation qui avait pour point central une très intéressante table ronde sur le thème «L'interprétariat sous toutes ses formes». Y participaient une interprète communautaire, une interprète de conférence, une médiatrice en gestion de conflits et une interprète en langue des signes (à l'intention de personnes sourdes). Ce qu'a dit cette dernière en particulier a été une découverte pour beaucoup des auditeurs. Il est apparu que les questions qui se posent, si elles ont bien entendu des spécificités selon les différents contextes, ont aussi des dimensions substantielles communes. Par exemple en ce qui concerne les principes déontologiques, notamment la fidélité, le secret professionnel et la neutralité.

L'interprétariat communautaire s'est surtout développé dans le passé récent. En Suisse, l'association Interpret, d'où émane l'article de Monika Joss, regroupe les personnes formées, qui viennent de passer le nombre de 500. A noter qu'il existe aussi une organisation au niveau international, Critical Link. Dans le domaine des soins et en un mot comme en cent, la disponibilité de compétences d'interprétariat est au- jourd'hui partie intégrante de la good medical practice. Or, il faut souligner l'insuffisance actuelle de leur prise en charge par des mécanismes collectifs (assurance-maladie, pouvoirs publics). Appartenances et d'autres reçoivent certaines subventions mais le remboursement de l'interprétariat reste un parent pauvre. C'est une lutte constante pour accréditer l'idée que, quand l'établissement d'une bonne relation thérapeutique n'est simplement pas possible sans la passerelle d'un interprète adéquatement formé, il n'est pas admissible de rester sans moyens de financer cette prestation - et ceci de façon régulière, sans qu'il doive s'agir de décisions exceptionnelles ou de cas en cas (cela vaut de la même manière pour les questions, souvent délicates, qui en milieu scolaire doivent être discutés avec des parents d'élèves allophones).

A ce propos, la responsable concernée d'Appartenances me signale la réaction négative d'un médecin qui se voit facturer le travail de l'interprète à laquelle il a eu recours pour expliquer à une patiente les enjeux vitaux d'une grossesse à haut risque. On peut comprendre ce praticien ... difficile de prétendre que c'est à lui personnellement d'assumer ce montant. Il est ainsi indispensable et urgent de sensibiliser (plus que cela, de convaincre) les financeurs usuels de prendre en charge ce service - et ceci dans le cadre de l'assurance de base, celles et ceux qui ont besoin d'interprètes sont souvent parmi les plus défavorisés.

Je profite de cette page pour dire aussi tout l'intérêt que j'ai trouvé à l'article d'Erhard Taverna sur notre confrère David Winizki et sa pratique au service de personnes venues d'ailleurs, pas rarement sans-papiers [2]. Une problématique qu'Appartenances connaît bien, par sa consultation psychothérapeutique pour migrants en particulier. Il est important de rendre visibles celles et ceux qui se préoccupent des personnes «invisibles» parmi nous; personnes qui vivent dans des situations difficiles, précaires, donnant trop souvent lieu à leur exploitation comme cela est relevé. Que je note enfin qu'il serait bien souhaitable que cet article soit disponible en français aussi!

Jean Martin 